\title{
Quantification of Mitral Regurgitation With MR Phase-Velocity Mapping Using a Control Volume Method
}

\author{
George P. Chatzimavroudis \\ Cleveland State University \\ John N. Oshinski \\ Emory University Hospital \\ Follow this and additional works at: https://engagedscholarship.csuohio.edu/encbe_facpub \\ Roderic I. Pettigrew

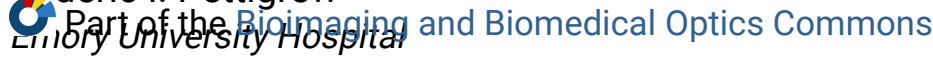 \\ How does access to this work benefit you? Let us know! \\ Reter /ishlikalkerstatement \\ University of Leeds \\ This is the accepted version of the following article: Chatzimavroudis GP, Oshinski JN, Pettigrew \\ Rphatker Frenteranch RH, Yoganathan AP. Quantification of mitral regurgitation with MR phase-

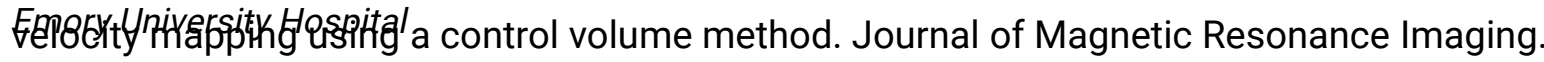 \\ 1998;8:577-582. , which has been published in final form at [http://onlinelibrary.wiley.com/doi/

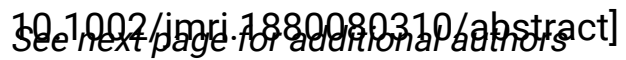

\section{Original Citation}

Chatzimavroudis GP, Oshinski JN, Pettigrew RI, Walker PG, Franch RH, Yoganathan AP. Quantification of mitral regurgitation with MR phase-velocity mapping using a control volume method. Journal of Magnetic Resonance Imaging. 1998;8:577-582.

\section{Repository Citation}

Chatzimavroudis, George P.; Oshinski, John N.; Pettigrew, Roderic I.; Walker, Peter G.; Franch, Robert H.; and Yoganathan, Ajit P., "Quantification of Mitral Regurgitation With MR Phase-Velocity Mapping Using a Control Volume Method" (1998). Chemical \& Biomedical Engineering Faculty Publications. 107.

https://engagedscholarship.csuohio.edu/encbe_facpub/107

This Article is brought to you for free and open access by the Chemical \& Biomedical Engineering Department at EngagedScholarship@CSU. It has been accepted for inclusion in Chemical \& Biomedical Engineering Faculty Publications by an authorized administrator of EngagedScholarship@CSU. For more information, please contact library.es@csuohio.edu. 


\section{Authors}

George P. Chatzimavroudis, John N. Oshinski, Roderic I. Pettigrew, Peter G. Walker, Robert H. Franch, and Ajit P. Yoganathan 


\title{
Quantification of Mitral Regurgitation With MR Phase- Velocity Mapping Using a Control Volume Method
}

\author{
George P. Chatzimavroudis, PhD - John N. Oshinski, PhD - Roderic I. Pettigrew, MD, PhD \\ Peter G. Walker, PhD • Robert H. Franch, MD - Ajit P. Yoganathan, PhD
}

Reliable diagnosis and quantification of mitral regurgitation are important for patient management and for optimizing the time for surgery. Previous methods have often provided suboptimal results. The aim of this in vitro study was to evaluate MR phase-velocity mapping in quantifying the mitral regurgitant volume (MRV) using a control volume (CV) method. A number of contiguous slices were acquired with all three velocity components measured. A CV was then selected, encompassing the regurgitant orifice. Mass conservation dictates that the net inflow into the $\mathrm{CV}$ should be equal to the regurgitant flow. Results showed that a CV, the boundary voxels of which excluded the region of flow acceleration and aliasing at the orifice, provided accurate measurements of the regurgitant flow. A smaller $\mathrm{CV}$ provided erroneous results because of flow acceleration and velocity aliasing close to the orifice. A large CV generally provided inaccurate results because of reduced velocity sensitivity far from the orifice. Aortic outflow, orifice shape, and valve geometry did not affect the accuracy of the $\mathrm{CV}$ measurements. The CV method is a promising approach to the problem of quantification of the MRV.

Index terms: Mitral regurgitation Control volume $\cdot$ Regurgitant volume Aortic outflow

JMRI 1998; 8:577-582

Abbreviations: $\mathrm{CV}=$ control volume, $\mathrm{MRV}=$ mitral regurgitant volume, PISA $=$ proximal isovelocity surface area, PVM $=$ phase-velocity mapping, $\mathrm{VENC}=$ velocity-encoding value
RELIABLE ASSESSMENT OF the severity of mitral re gurgitation is important for patient management and for determining the time for valve replacement surgery. To date, there are a number of clinical approaches to diagnosing the severity of valvular regurgitation. Unfortunately, these approaches usually lack reliability. Angiography (1) has been the standard clinical technique, but it is invasive, only semiquantitative, and involves radiation exposure. A variety of echocardiographic and MRI techniques (2-10) have been studied recently to assess mitral regurgitation. They focused on the jet side of the regurgitant valve in an attempt to correlate the geometrical parameters of the regurgitant jet in the left atrium with the severity of the disease. Unfortunately, these techniques were accompanied by technical limitations. Lately, efforts toward quantifying the regurgitant volume have been increased. This quantity has been identified as an important index of the severity of regurgitation. Vol umetric MRI techniques $(11,12)$ attempted to measure the regurgitant volume from the difference between the left and right ventricular stroke volumes. Although the combination of left and right ventricular volumes with systolic and diastolic aortic and pulmonic flows can provide an estimate for the regurgitant volumes of the heart valves, this approach is not reliable in the presence, for instance, of aortic and pulmonic valve stenosis because of signal distortion in the aorta and pulmonary artery. Furthermore, volumetric MRI techniques indirectly estimate the regurgitant volume by combining measurements at different sites in the heart and not focusing on the site of interest, which is the specific regurgitant valve. The recently developed proximal isovelocity surface area (PISA) method (13) seems promising because it attempts to focus solely on the mitral valve. However, its reliability and accuracy can be severely affected by the complex nature of the flow field in the left ventricle because of the interaction of the regurgitant flow field with the aortic outflow field (14). The dominant aortic outflow field contaminates the proximal accelerating regurgitant flow field, altering the isovelocity contour shape and causing errors in the calculation of the regurgitant flow rate.

Using MR phase-velocity mapping (PVM) flow through a vessel can be measured accurately (15-18). PVM has 
been used to measure the mitral regurgitant volume in an indirect way, by subtracting the aortic outflow measured in systole from the mitral inflow measured in diastole (19). One of the disadvantages of this indirect method is that it is not valid in the simultaneous presence of mitral and aortic regurgitation.

In aortic regurgitation, the regurgitant flow rate can be measured with PVM by positioning an imaging slice perpendicular in the aorta close to the aortic valve (18). Integration of the aortic flow curve over diastole provides the regurgitant volume per cardiac cycle. Under the current imaging technology, this single slice technique is inaccurate in mitral regurgitation, because of the strong interaction between the aortic outflow and the regurgitant flow in the vicinity of the mitral valve (Fig. 1).

In a recent study, Walker et al (20) showed that flow through an orifice can be measured with a control volume (CV) method using PVM. Therefore, the idea in this study is to take a number of contiguous imaging slices in the vicinity of the mitral valve model and measure all three velocity components. Then, an imaginary $\mathrm{CV}$ is constructed to encompass the orifice. Because three-dimensional velocity data are available in all slices, it is easy to select a rectangular box $(\mathrm{CV})$ in which the faces consist of voxels from these slices. One of the six faces of this box will cut the regurgitant orifice parallel to the orifice plane. Because of the principle of mass conservation, the net inflow into this box or CV (excluding the voxels inside the orifice) is equal to the regurgitant flow through the orifice.

Therefore, the hypothesis in this study was that this $\mathrm{CV}$ method has the potential to measure the mitral regurgitant volume. However, there is a need to determine the effect of a variety of factors on the reliability of the method to quantify the regurgitant volume. The aim of this study was to conduct an in vitro investigation for the potential of the $\mathrm{CV}$ method to accurately quantify the mitral regurgitant volume. Specifically, we investigated the effects of the size of the CV, the presence of aortic outflow, and the geometrical characteristics of the regurgitant orifice on the reliability and accuracy of the CV measurements.

\section{- METHODS}

\section{Models}

A plexiglas left ventricular model (Fig. 2) with the aortic outflow tract was used for the experiments. The "baseapex" size was $7.6 \mathrm{~cm}$, and the "anterior-posterior" size was $7.0 \mathrm{~cm}$. The model was designed to allow coneshaped plexiglas regurgitant mitral valve models to be inserted easily. Seven of these models were made to study different magnitudes of regurgitant flow rates and different geometries. Four of the models had a circular regurgitant orifice. Two of these circular orifice models had a cone-apex angle of $60^{\circ}$ and the other two had an angle of $90^{\circ}$. For each angle, the orifice diameters used were $3 \mathrm{~mm}$ and $5 \mathrm{~mm}$. The other three models had slit-like orifices with length to width ratios of $2.5: 1,5: 1$, and 10:1. The model was placed in a plexiglas vessel filled with stationary water to ensure an adequate MR signal.

\section{Measurements}

Steady and pulsatile flow experiments were performed in a 1.5-T scanner (Gyroscan ACS II, Philips Medical Systems, Shelton, CT). Water was used as the working fluid, because the flow in the model was inertially driven. Use of steady flow allowed the factors of interest to be investi-

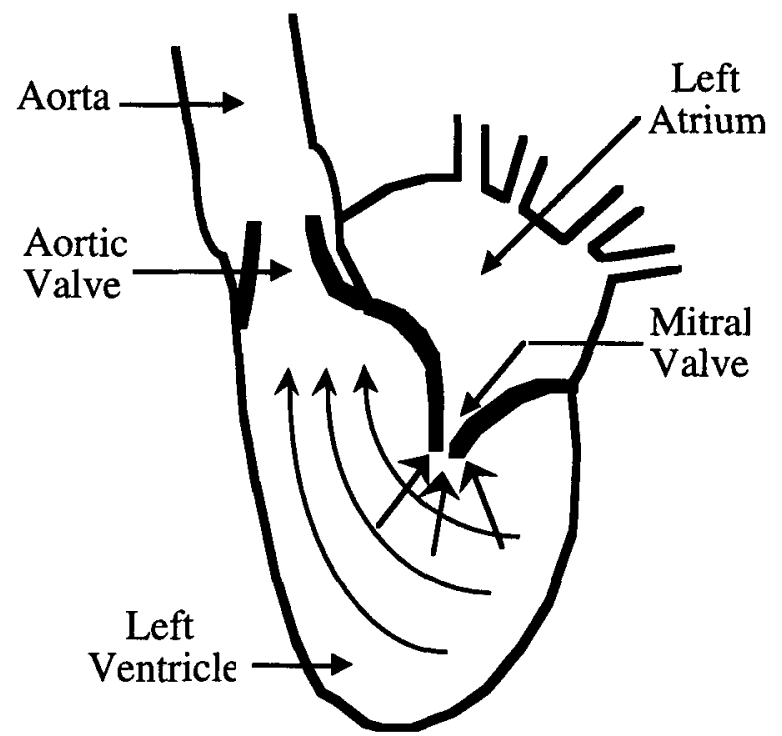

Figure 1. Left heart and mitral regurgitation. In systole, the regurgitant flow interacts with the aortic outflow, which is ejected into the aorta.

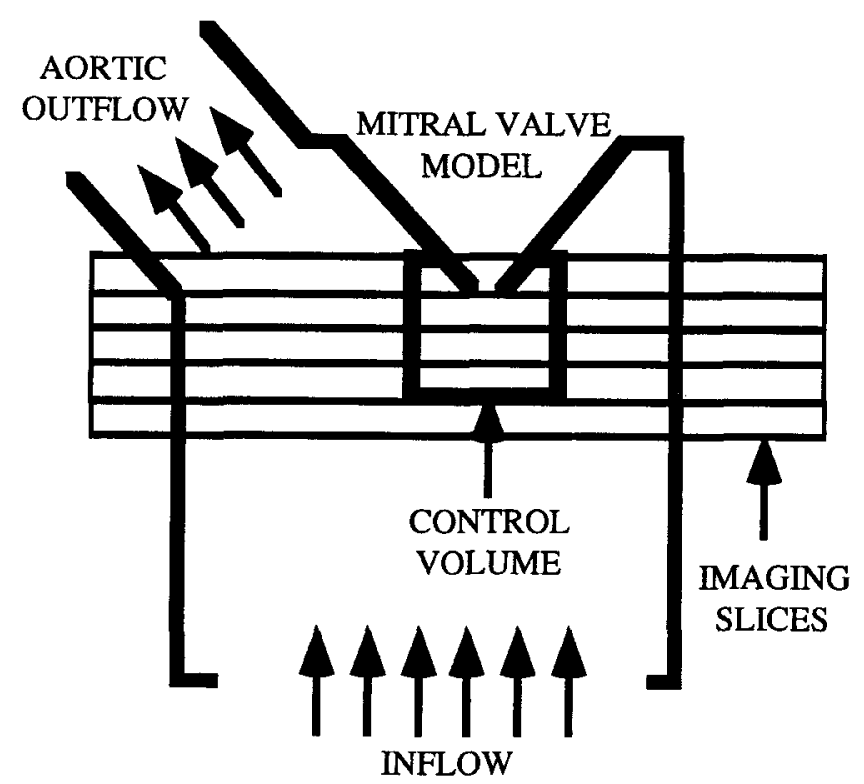

Figure 2. In vitro model of the left ventricle used in the experiments. A number of contiguous imaging slices were acquired at the valve region. From the three-dimensional velocity data, an imaginary three-dimensional rectangular $\mathrm{CV}$ was constructed to encompass the orifice.

gated separately. Finally, pulsatility was introduced to study the method under physiologic flow conditions.

In the steady flow experiments, the regurgitant flow rate was varied from 1.0 to $7.01 / \mathrm{min}$ and the aortic outflow rate was varied between 0 and $251 / \mathrm{min}$ (Table 1 ). These flow rates were measured during the experiments with calibrated rotameters. Flow was driven by a steady flow pump (4E-34N, Little Giant Pump Company, Oklahoma City, OK).

In the pulsatile flow experiments, the regurgitant volume was varied between 10 and $55 \mathrm{ml} /$ beat (Table 2), covering a large range of regurgitant severity. The aortic outflow was kept constant to $70 \mathrm{ml} /$ beat in all cases. Pulsatility in the flow was generated by a computer-controlled piston pump (SuperPump, SPS 3891 Vivitro Systems Inc., Victoria, British Columbia, Canada) able to reproduce physio- 


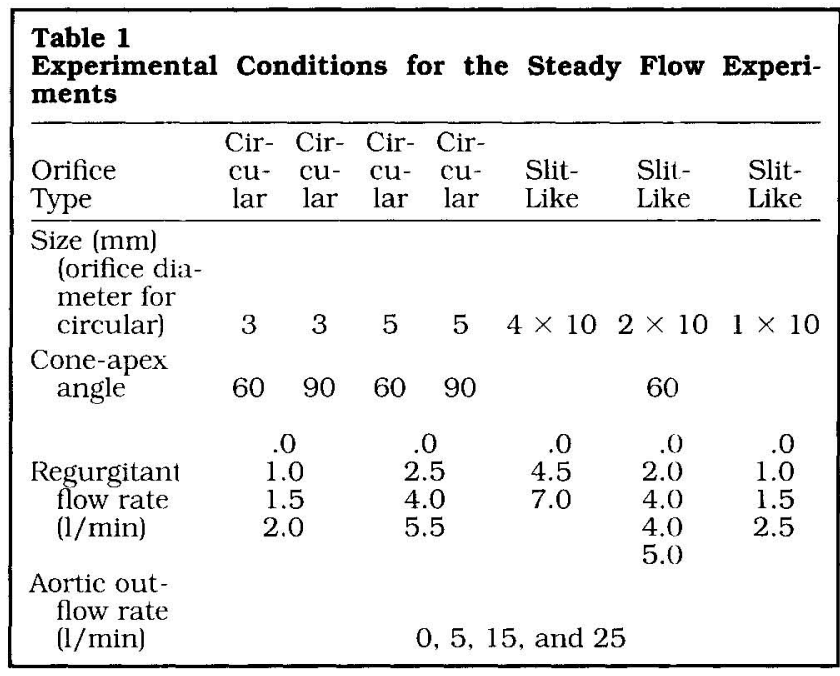

\begin{tabular}{|c|c|c|c|c|c|c|}
\hline \multirow{2}{*}{$\begin{array}{l}\text { Orifice } \\
\text { Type } \\
\text { Size (mm) } \\
\text { (orifice dia- } \\
\text { meter for } \\
\text { circular) }\end{array}$} & $\begin{array}{l}\text { Cir- } \\
\text { cu- } \\
\text { lar }\end{array}$ & $\begin{array}{l}\text { Cir- } \\
\text { cu- } \\
\text { lar }\end{array}$ & \multicolumn{2}{|c|}{$\begin{array}{l}\text { Slit- } \\
\text { Like }\end{array}$} & \multicolumn{2}{|c|}{$\begin{array}{l}\text { Slit- } \\
\text { Like }\end{array}$} \\
\hline & 3 & 5 & \multicolumn{2}{|c|}{$4 \times 10$} & \multicolumn{2}{|c|}{$2 \times 10$} \\
\hline $\begin{array}{l}\text { Cone-apex } \\
\text { angle }\end{array}$ & 90 & 60 & \multicolumn{4}{|c|}{60} \\
\hline $\begin{array}{l}\text { Regurgitant } \\
\text { volume } \\
\text { (ml/cycle) }\end{array}$ & 10 & 40 & 35 & 55 & 20 & 37 \\
\hline $\begin{array}{l}\text { Peak regurgitant } \\
\text { orifice velocity } \\
(\mathrm{m} / \mathrm{sec})\end{array}$ & 4.7 & 6.8 & 3.9 & 5.0 & 3.5 & 6.8 \\
\hline Rate (cycles/min) & \multicolumn{6}{|c|}{60} \\
\hline $\begin{array}{l}\text { Net "cardiac" } \\
\text { output }(1 / \mathrm{min})\end{array}$ & \multicolumn{6}{|c|}{4.2} \\
\hline
\end{tabular}

logic flow waveforms. The flow waveforms were measured with an MRI-compatible $24 \mathrm{~N}$ in-line brass transit time ultrasonic flow probe (Transonic Systems Inc., Ithaca, NY), using a single channel T-108 flowmeter (Transonic Systems Inc., Ithaca, NY). The flow waveform was recorded on a Macintosh IIci (Apple Computers Inc., Cupertino, CA) computer by digitizing the analog signal from the flowmeter with an A/D board (NB-MIO-16, National Instruments Inc., Austin, TX), using the LabVIEW software (Version 3.0, National Instruments Inc.).

\section{Imaging Procedure}

Initially, a gradient-echo scout image was acquired, as shown in Figure 3 (slice thickness $=7 \mathrm{~mm}$, field of view $=300 \mathrm{~mm}, \mathrm{TE}=8 \mathrm{msec}, \mathrm{TR}=30 \mathrm{msec}$, flip angle $=35^{\circ}$, matrix size $=256 \times 256$ ). The mitral regurgitant orifice was detected on this scout image, and then five contiguous transverse velocity-encoded images, with all three velocity components measured, were acquired in the region of the regurgitant orifice (Figs. 1 and 3), perpendicular to the transvalvular axis (slice thickness $=5 \mathrm{~mm}$; field of view $=$ $300 \mathrm{~mm} ; \mathrm{TE}=7.2,5.4$, and $6.9 \mathrm{msec}$ for the throughplane, anterior-posterior, and left-right velocity compo-

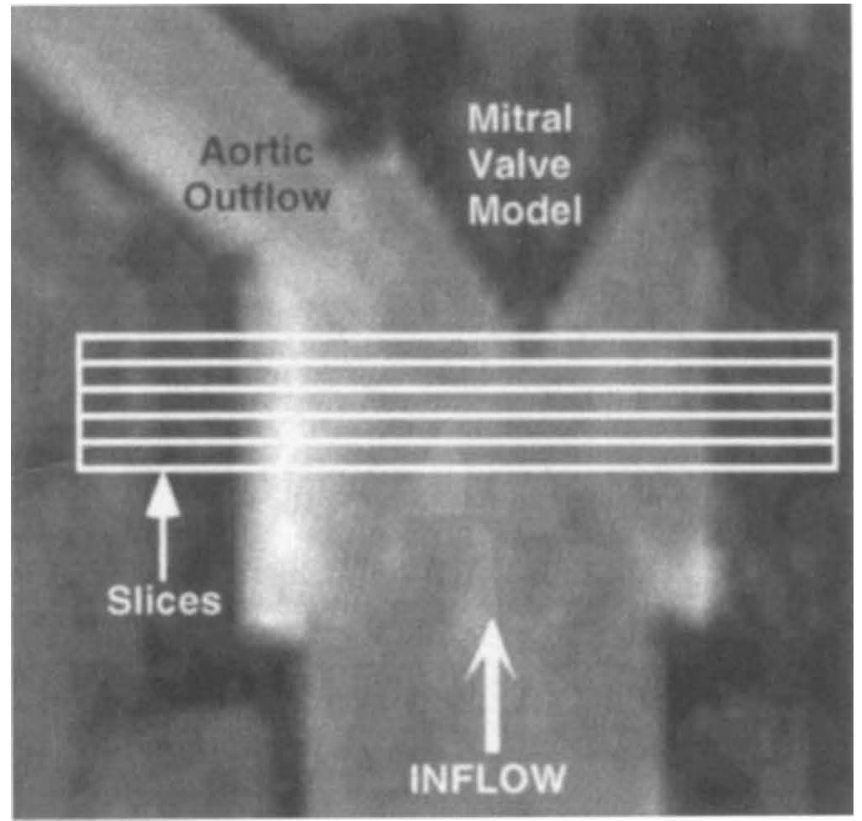

Figure 3. Sagittal gradiert-echo scout image of the mitral model. The mitral regurgitant orifice was located and five transverse slices were placed in the vicinity of this orifice.

nent, respectively; TR $=30$ msec; matrix size $=128 \times$ 128; flip angle $=35^{\circ}$ ). The spatial resolution of the images (voxel size) was $5 \times 2.34 \times 2.34 \mathrm{~mm}$. Although the inplane resolution could be higher, increasing this resolution would cause an additional increase in the already long acquisition time. Because preliminary measurements with the $2.34 \times 2.34 \mathrm{~mm}$ resolution provided accurate results, it was decided to perform the main measurements with this value. Based on preliminary measurements, the velocity-encoding value (VENC) used in the main experiments was $45 \mathrm{~cm} / \mathrm{sec}$. Use of this value prevented velocity aliasing in the region of interest in the vicinity of the regurgitant orifice. In pulsatile flow, each velocity acquisition was performed with retrospective ECG gating. Twenty phases were acquired during the cycle.

\section{Image and Data Analysis}

Based on the phase values of the pixels in the stationary water surrounding the model, a plane containing the phase offsets was created through two-dimensional regression analysis, as previously described in detail $(18,21)$. Subtraction of this error plane from each initial phase image resulted in a series of new corrected phase images. Subsequently, the phase was converted to velocity based on the linearity between the phase of the received signal and the protons velocity. A computer program was used to select a series of rectangular CVs that encompassed the regurgitant orifice (Figs. 2 and 4). The range of rectangular $\mathrm{CV}$ sizes was from $7 \times 7 \times 7.5$ $\mathrm{mm}$ to $40 \times 40 \times 22.5 \mathrm{~mm}$ (length $\times$ width $\times$ height). Integration of the velocity over the area of the faces of the CV (except for the pixels located on the mitral model wall and inside the regurgitant orifice) provided the net flow rate that entered the CV. Due to mass conservation, this flow rate should be equal to the regurgitant flow rate. In pulsatile flow, the regurgitant volume was determined by integrating the regurgitant flow curve over systole.

\section{Statistical Analysis}

The measured flow rates were compared to the actual flow rates with regression analysis. This analysis was 


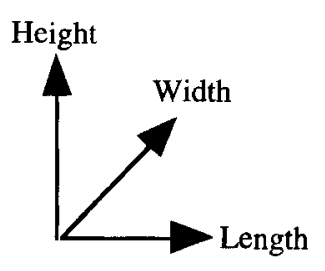

- - - - . Slices' Boundaries

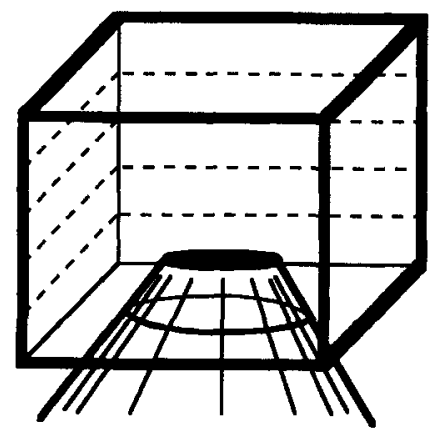

Mitral Valve Model

Figure 4. Geometric characteristics of CV. Height corresponds to the base-apex direction ( $z$ or foot-head direction in the scanner); length corresponds to the anterior-posterior (or $x$ in the scanner) direction; and width corresponds to the left-right (or $y$ in the scanner) direction.

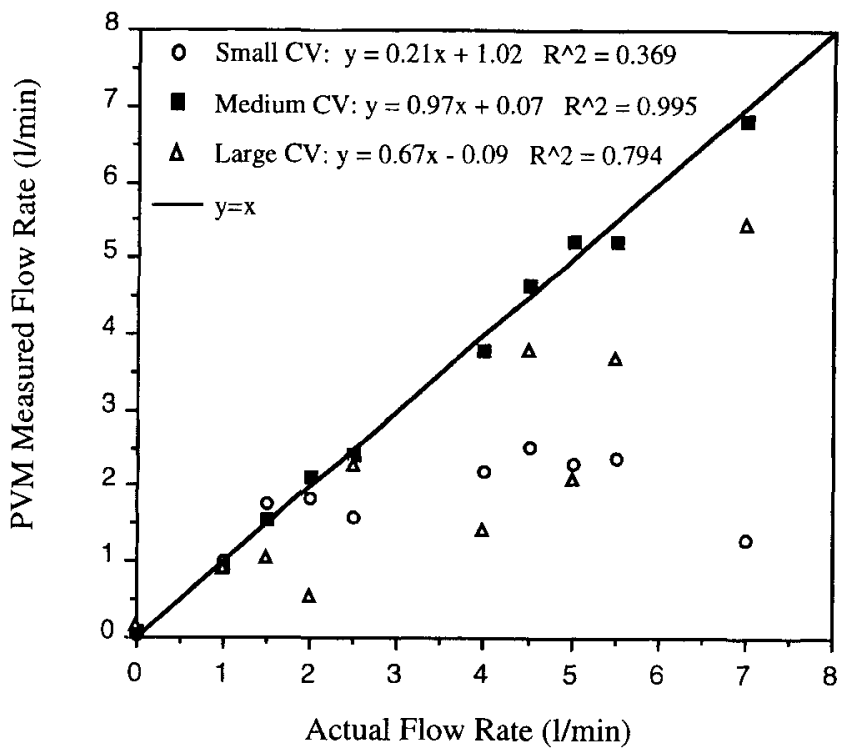

Figure 5. Relationship between the CV measured regurgitant flow rate and the actual regurgitant flow rate for small, medium, and large $\mathrm{CV}$ sizes.

performed for different CV sizes to determine the size range that provided the most accurate results. Nonparametric Mann-Whitney statistical tests were used to determine the effects of aortic outflow and geometry of the orifice models.

\section{- RESULTS}

In Figure 5, the relation between the CV measured regurgitant flow rate and the actual regurgitant flow rate is shown. Results for three CV sizes are shown: small $(7 \times$ $7 \times 7.5 \mathrm{~mm})$, medium $(26 \times 26 \times 17.5 \mathrm{~mm})$, and very large $(40 \times 40 \times 22.5 \mathrm{~mm})$. For the small $\mathrm{CV}$, the flow rate was measured satisfactorily only for very small flow rates. An underestimation in the measurements was seen as the regurgitant flow rate increased. Regression analysis confirmed the previous observations as seen from the regression equation. For the medium $\mathrm{CV}$, the measured flow rates are in very good agreement with the true flow rates for the entire range of flow rates. This was confirmed by the regression equation. Use of a very large $\mathrm{CV}$ leads to underestimation of the flow rate and scattering in the data (reduced coefficient of determination).

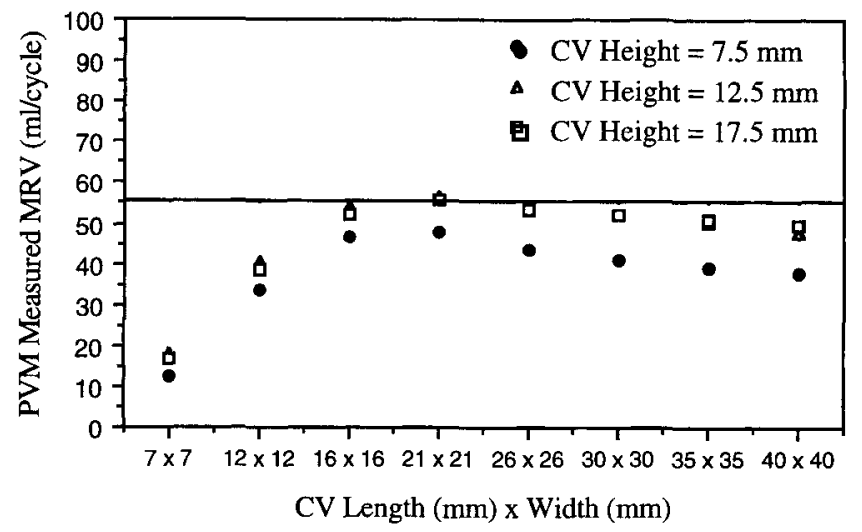

Figure 6. Measured MRV with the $\mathrm{CV}$ method for an actual regurgitant volume of $55 \mathrm{ml} /$ cycle.

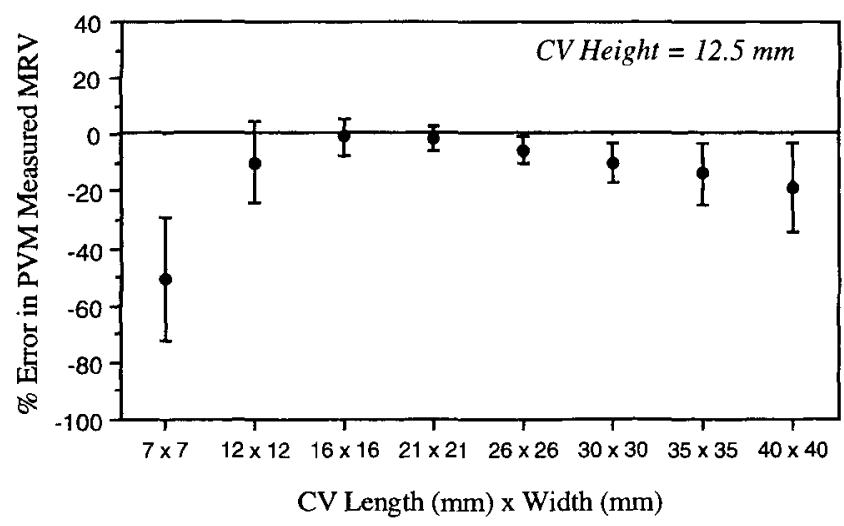

Figure 7. Average percent error in the measured regurgitant volume for different $\mathrm{CV}$ length and width and for a CV height of three slices.

The same behavior was seen in the pulsatile flow measurements. A case of severe regurgitation is shown in Figure 6 . Use of a small CV $(7 \times 7 \times 7.5 \mathrm{~mm})$ led to errors in the measurements. The accuracy was very good for a medium CV $(21 \times 21 \times 12.5 \mathrm{~mm})$. In Figure 7 , the average percent error in the measured regurgitant volume is shown plotted against the $\mathrm{CV}$ length and width when the CV height is $12.5 \mathrm{~mm}$ (three slices). The variability in the measurements for each case can be seen from the error bars. In the case of small $(<16 \mathrm{~mm})$ or large $1>26$ $\mathrm{mm}$ ) CV length and width, the variability is high and the reliability of the measurements is smaller compared to a medium CV size, in which both the mean errors and variability are small.

In Figure 8, no significant difference can be observed in the regurgitant volume results under different magnitudes of the aortic outflow $(0-251 / \mathrm{min})$. In all cases, even with no aortic outflow, best accuracy was observed for a medium CV (16-26 mm) length and width. The accuracy was reduced too close or too far from the orifice. Statistical analysis with a Mann-Whitney test confirmed the similarity of the results regardless of the aortic outflow rate ( $P$ values from . 18 to .87).

No significant difference was observed in the results between the circular and the slit-like orifice shapes. This was also confirmed by statistical analysis with a MannWhitney test ( $P$ values from .37 to .96 ). Similarly, no significant difference was observed in the trends of the data for a $60^{\circ}$ and a $90^{\circ}$ cone-apex angle of the mitral valve models. Statistical analysis with a Mann-Whitney test confirmed the similarity in the measurements $(P>.20)$. 


\section{- DISCUSSION}

The main objective of this study was to investigate the potential of the $\mathrm{CV}$ method to quantify the mitral regurgitant volume and to study the effects of CV size, aortic outflow, and geometrical characteristics of the regurgitant valve on the accuracy of the measurements. Both steady and pulsatile flow data indicated that the $\mathrm{CV}$ should be selected carefully.

By selecting a very small $\mathrm{CV}$, its boundary voxels contained distorted signal caused by rapid convective flow acceleration $(22,23)$ or velocity aliasing. The peak orifice velocity in mitral regurgitation can reach or even exceed $5 \mathrm{~m} / \mathrm{sec}$. Even if a VENC of this value is chosen to avoid velocity aliasing, acceleration will still cause either signal loss or overestimation (in moderate cases) of the actual velocity in that region. By choosing a larger $\mathrm{CV}$ in which the boundary voxels were not in the region of acceleration or aliasing, accurate measurements of the regurgitant flow were obtained. Because the velocity decreases very rapidly with the distance from the orifice, a VENC much smaller than $5 \mathrm{~m} / \mathrm{sec}$ must be chosen to optimize the measurements.

When the $\mathrm{CV}$ was very large, its boundary voxels were in regions with velocities much lower than the VENC selected. The velocity sensitivity was low, resulting in inaccurate measurements. By choosing a $\mathrm{CV}$ of a size large enough to exclude the region of disturbed flow near the regurgitant orifice, the velocity sensitivity was high enough and the measurements were accurate.

Aortic outflow did not affect the accuracy of the measurements. This is an important finding, considering that the presence of aortic outflow is one of the limitations of the color Doppler PISA technique (14). It is the ability of PVM to measure all three velocity components in a slice and the theoretical simplicity of this CV method that provide this accuracy in the presence of aortic outflow.

The accuracy in the results of the regurgitant volume, regardless of the shape of the regurgitant orifice or the angle between the valve leaflets (simulated through the examination of two cone-apex angles), shows that geometrical characteristics of the mitral valve should not affect the reliability of the $\mathrm{CV}$ method in measuring the regurgitant volume. This is a result of the nature of the CV method, which does not require any assumptions about the converging flow field (in contrast to the PISA method).

The present study used a simple in vitro ventricular model with cone-shaped mitral valve models and an aortic outflow tract. The general geometry was similar to that of a human left ventricle with regard to flow patterns in the ventricle, valve shape, and the relative position of the mitral valve and the aortic outflow track. MR images showed that an interaction existed between the aortic outflow and the regurgitant flow (Fig. 9), similar to that observed from Doppler in vivo images. Of course, this model did not exactly represent the human left ventricle, because there were functional and geometrical physiologic deviations. For example, no wall motion was simulated in the experiments. However, in this study, it was of interest to investigate whether the CV method is reliable in a flow environment similar to that observed clinically in patients with mitral regurgitation, in which aortic outflow interacts with the regurgitant flow. Other factors, such as the ventricular wall motion and the mitral valve motion during systole, should be investigated next in the course of establishment of this method clinically to measure the mitral regurgitant volume.

The major current limitation of this approach is its long acquisition time. For instance, acquiring four slices, with

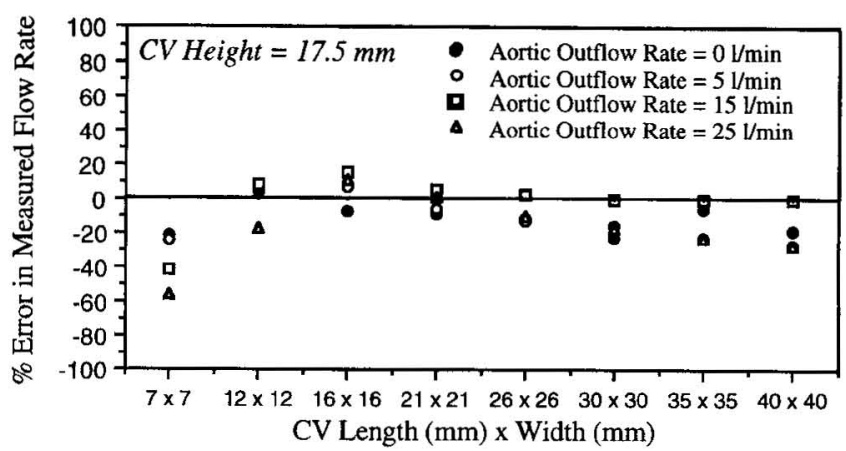

Figure 8. Comparison between measurements of the regurgitant flow rate for different magnitudes of the aortic outflow.

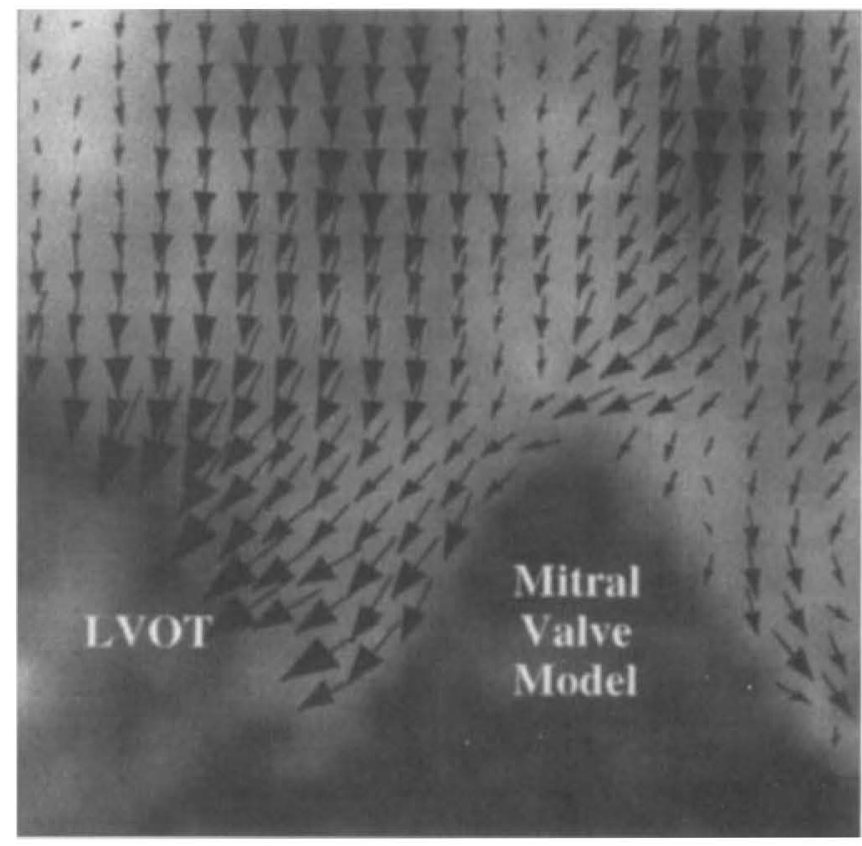

Figure 9. Visualization of the flow field close to the mitral regurgitant orifice. LVOT = left ventricular outflow tract.

all three velocity components measured in each slice, required more than 1 hour of imaging. It should be pointed out that conventional pulse sequences were used in this study. Use of turbo gradient-echo, echo-planar flow imaging, and other ultrafast techniques $(24,25)$ will provide short imaging times. These techniques are currently under investigation regarding their reliability and accuracy to measure blood velocity with promising results. The aim of this study was to show that the CV method is accurate using a well calibrated and extensively used pulse sequence.

Although the $\mathrm{CV}$ method is very promising in quantifying the regurgitant volume, mitral regurgitation is a complex disease. Several indices must be known before complete diagnosis is done. Knowledge of the regurgitant volume is important, because it provides an index of the severity of the disease. It can also be very useful in monitoring the effectiveness of drugs, such as vasodilators, used to treat patients with regurgitation and in following patients. This quantitative information combined with information regarding the condition and function of the heart that MRI provides can lead to a more complete cardiac evaluation.

In conclusion, in vitro measurements showed that the $\mathrm{CV}$ method is very promising for the quantification of the mitral regurgitant volume. The $\mathrm{CV}$ size must be selected 
carefully to be large enough for the boundary voxels to exclude the region of flow acceleration and velocity aliasing close to the regurgitant orifice. The presence of aortic outflow and the shape of the regurgitant orifice or the geometry of the valve do not affect the reliability and accuracy of the PVM measurements.

\section{References}

1. Croft $\mathrm{CH}$, Lipscomb K, Mathis K, et al. Limitations of qualitative angiographic grading in aortic or mitral regurgitation. Am J Cardiol 1984; 53:1593-1598.

2. Ciobanu M, Abbasi AS, Allen M. Hermer A, Spellberg R. Pulsed Doppler echocardiography in the diagnosis of severity of aortic regurgitation. Am J Cardiol 1982; 49:339-343.

3. Veyrat C, Ameur A, Gourtchiglouian C, Lessana A, Abitbol G, Kalmanson D. Calculation of pulsed Doppler left ventricular outflow tract regurgitant index for grading the severity of aortic regurgitation. Am Heart $J$ 1984: 108:507-515.

4. Holm S, Eriksson P, Karp K. Osterman G, Teien D. Quantitative assessment of aortic regurgitation by combined two-dimensional, continuous-wave and colour flow Doppler measurements. J Intern Med 1992; 231:115-121.

5. Perry GJ, Helmcke F, Nanda NC, Byard C, Soto B. Evaluation of aortic insufficiency by Doppler color flow mapping. J Am Coll Cardiol 1987; 9:952-959.

6. Wagner S, Auffermann W, Buser P, et al. Diagnostic accuracy and estimation of the severity of valvular regurgitation from the signal void on cine magnetic resonance images. Am Heart $J$ $1989 ; 118: 760-767$

7. Suzuki J-I, Caputo GR, Kondo C, Higgins CB. Cine MR imaging of valvular heart disease: display and imaging parameters affect the size of the signal void caused by valvular regurgitation. Am J Roentgenol 1990; 155:723-727.

8. Taylor AL, Eichhorn EJ, Brickner ME, Eberhart RC, Grayburn PA. Aortic valve morphology: an important in vitro determinant of the proximal regurgitant jet width by Doppler color flow mapping. J Am Coll Cardiol 1990; 16:405-412.

9. Reynolds T, Abate J, Tenney A, Warner MG. The JH/LVOH method in the quantification of aortic regurgitation: how the cardiac sonographer may avoid an important potential pitfall. J Am Soc Echocardiogr 1991; 4:105-108.

10. Globits S. Mayr H, Neuhold A, Globar D. Quantitative assessment of aortic regurgitation by magnetic resonance imaging. Eur Heart J 1992; 13:78-83.

11. Sechtem U, Pflugfelder PW, Cassidy MM, et al. Mitral or aortic regurgitation: quantification of regurgitant volumes with cine MR imaging. Radiology 1988; 167:425-430.
12. Dulce M-C, Mostbeck GH, O'Sullivan M, Cheitlin M, Caputo GR, Higgins CB. Severity of aortic regurgitation: interstudy reproducibility of measurements with velocity-encoded cinc MR imaging. Radiology 1992; 185:234-240.

13. Recusani F, Bargiggia GS, Yoganathan AP, et al. A new method for quantification of regurgitant flow rate using color Doppler flow imaging of the flow convergence region proximal to $\hat{a}$ discrete orifice. Circulation 1991; 83:594-604.

14. Hopmeyer J, Fontaine AA, Yang A, Levine RA, Yoganathan AP. The effect of aortic outflow on the quantification of mitral regurgitation using the flow convergence method. J Am Soc Echocardiogr 1996; 9:44-57.

15. Firmin DN, Nayler GL, Klipstein RH, Underwood SR, Rees RSO, Longmore DB. In vivo validation of MR velocity imaging. $J$ Comput Assist Tomogr 1987; 11(5):751-756.

16. Meier D, Maier S, Bosiger P. Quantitative flow measurements on phantoms and on blood vessels with MR. Magn Reson Med $1988 ; 8: 25-34$.

17. Kilner PJ, Yang GZ, Mohiaddin RH, Firmin DN, Longmore DB. Helical and retrograde secondary flow patterns in the aortic arch studied by three-directional magnetic resonance velocity mapping. Circulation 1993; 88:2235-2247.

18. Chatzimavroudis GP. Walker PG. Oshinski JN, Franch RH. Pettigrew RI, Yoganathan AP. Slice location dependence of aortic regurgitation measurements with MR phase velocity mapping. Magn Reson Med 1997; 37:545-551.

19. Fujita A. Chazouilleres AF, Hartiala JJ, et al. Quantification of mitral regurgitation by velocity-encoded cine nuclear magnetic resonance imaging. Am J Cardiol 1994; 23:951-958.

20. Walker PG, Oyre S, Pedersen EM, Houlind K, Guenet FSA, Yoganathan AP. A new control volume method for calculating valvular regurgitation. Circulation 1995; 92:579-586.

21. Walker PG, Cranney GB, Scheidegger MB, Waseleski G, Pchost GM, Yoganathan AP. Semiautomated method for noise reduction and background phase error correction in MR phase velocity data. JMRI 1993: 3:521-530.

22. Oshinski JN, Ku DN, Bohning DE, Pettigrew RI. Effects cf acceleration on the accuracy of MR phase velocity measurements. JMRI 1992: 2:665-670.

23. Kouwenhoven M, Hofman MBM, Sprenger M. Motion induced phase shifts in MR: acceleration effects in quantitative flow measurements - a reconsideration. Magn Reson Med 1995; 33 766-777.

24. Gatehouse PD, Firmin DN, Collins S, Longmore DB. Real time blood flow imaging by spiral scan phase velocity mapping. Magn Reson Med 1994; 31:504-512.

25. McKinnon GC, Debatin JF, Wetter DR, von Schulthess GK Interleaved echo planar flow quantification. Magn Reson Med 1994; 32:263-267. 